\title{
Zeroth-order randomized block methods for constrained minimization of expectation-valued Lipschitz continuous functions
}

\author{
Uday V. Shanbhag
}

\author{
Farzad Yousefian
}

\begin{abstract}
We consider the minimization of an $L_{0}$-Lipschitz continuous and expectation-valued function, denoted by $f$ and defined as $f(\mathbf{x}) \triangleq \mathbb{E}[\tilde{f}(\mathbf{x}, \omega)]$, over a Cartesian product of closed and convex sets with a view towards obtaining both asymptotics as well as rate and complexity guarantees for computing an approximate stationary point (in a Clarke sense). We adopt a smoothing-based approach reliant on minimizing $f_{\eta}$ where $f_{\eta}(\mathbf{x}) \triangleq \mathbb{E}_{u}[f(\mathbf{x}+\eta u)], u$ is a random variable defined on a unit sphere, and $\eta>0$. In fact, it is observed that a stationary point of the $\eta$-smoothed problem is a $2 \eta$-stationary point for the original problem in the Clarke sense. In such a setting, we derive a suitable residual function that provides a metric for stationarity for the smoothed problem. By leveraging a zeroth-order framework reliant on utilizing sampled function evaluations implemented in a block-structured regime, we make two sets of contributions for the sequence generated by the proposed scheme. (i) The residual function of the smoothed problem tends to zero almost surely along the generated sequence; (ii) To compute an $\mathrm{x}$ that ensures that the expected norm of the residual of the $\eta$-smoothed problem is within $\epsilon$ requires no greater than $\mathcal{O}\left(\frac{1}{\eta \epsilon^{2}}\right)$ projection steps and $\mathcal{O}\left(\frac{1}{\eta^{2} \epsilon^{4}}\right)$ function evaluations. These statements appear to be novel and there appear to be few results to contend with general nonsmooth, nonconvex, and stochastic regimes via zeroth-order approaches.
\end{abstract}

\section{INTRODUCTION}

We consider the following stochastic optimization problem

$$
\begin{array}{rl}
\min _{\mathbf{x}} & f(\mathbf{x}) \triangleq \mathbb{E}[\tilde{f}(\mathbf{x}, \xi(\omega))] \\
\text { subject to } & \mathbf{x} \in \mathcal{X} \triangleq \prod_{i=1}^{b} \mathcal{X}_{i},
\end{array}
$$

where $f: \mathbb{R}^{n} \rightarrow \mathbb{R}$ is a real-valued, nonsmooth, and nonconvex function, $\mathcal{X}_{i} \subseteq \mathbb{R}^{n_{i}}$ is a closed and convex set for $i=1, \ldots, b$ with $\sum_{i=1}^{b} n_{i}=n, \xi: \Omega \rightarrow \mathbb{R}^{d}$ denotes a random variable associated with the probability space $(\Omega, \mathcal{F}, \mathbb{P})$. Throughout, we assume that $f$ is $L_{0^{-}}$ Lipschitz continuous on the set $\mathcal{X}$, i.e., there exists a scalar $L_{0}>0$ such that for all $\mathbf{x}, \mathbf{y} \in \mathcal{X}$ we have $|f(\mathbf{x})-f(\mathbf{y})| \leq L_{0}\|\mathbf{x}-\mathbf{y}\|$. Further, at any $\mathbf{x} \in \mathbb{R}^{n}$, $f(\mathbf{x}) \triangleq \mathbb{E}[\tilde{f}(\mathbf{x}, \omega)]$ where we refer to $f(\mathbf{x}, \xi(\omega))$ by $f(\mathbf{x}, \omega)$.

While there is a significant body of literature on contending with nonsmooth stochastic convex optimization problems [19], most nonconvex generalizations are generally restricted to structured regimes where the nonconvexity often emerges as an expectation-valued smooth function while the

Industrial \& Manufacturing Eng., Pennsylvania State University, udaybagepsu.edu; Shanbhag acknowledges the support from NSF CMMI-1538605 and DOE ARPA-E award DE-AR0001076.

School of Industrial Eng. \& Management, Oklahoma State University, farzad.yousefian@okstate. edu; Yousefian acknowledges the support of the NSF through CAREER grant ECCS-1944500. nonsmoothness arises in a deterministic form . However, in many applications, $\tilde{f}(\bullet, \omega)$ may be both nonconvex and nonsmooth and proximal stochastic gradient schemes [10], [14] cannot be directly adopted. We now discuss some relevant research in nonsmooth and nonconvex regimes.

(a) Nonsmooth nonconvex optimization. In [1], Burke et al. disuss how gradient sampling allow for approximating the Clarke subdifferential of a function that is differentiable almost everywhere. A robust gradient sampling scheme was subsequenly developed [2] for functions that are continuously differentiable on an open subset $D \subseteq \mathbb{R}^{n}$; such functions need not be convex nor locally Lipschitz. In [2], the authors prove that a limit point of a subsequence is an $\epsilon$-Clarke stationary point when $f$ is locally Lipschitz while Kiwiel proved that every limit point is Clarke stationary with respect to $f$ without requiring compactness of level sets [13]. There have also been efforts to develop statements in structured regimes where $f$ is either weakly convex [7], [8] or $f=g+h$ and $h$ is smooth and possibly nonconvex while $g$ is convex, nonsmooth, and proximable [14], [21].

(b) Nonsmooth nonconvex stochastic optimization. Much of the efforts in the regime of stochastic nonconvex optimization have been restricted to structured regimes where $f(\mathbf{x})=h(\mathbf{x})+g(\mathbf{x}), h(\mathbf{x}) \triangleq \mathbb{E}[F(\mathbf{x}, \omega)], h$ is smooth and possibly nonconvex while $g$ is closed, convex, and proper with an efficient proximal evaluation. In such settings, proximal stochastic gradient techniques [10] and their variancereduced counterparts [10], [14] were developed.

(c) Zeroth-order methods. Deterministic [3] and randomized smoothing [9], [20] have been the basis for resolving a broad class of nonsmooth optimization problems [15], [22]. When the original function is nonsmooth and nonconvex, Nesterov and Spokoiny [17] examine unconstrained nonsmooth and nonconvex optimization problems via Gaussian smoothing.

Motivation. Our work draws motivation from the recent work by Zhang et al. [24] where the authors show that for a suitable class of nonsmooth functions, computing an $\epsilon$ stationary point is impossible in finite time. This negative result is a consequence of the possibility that the gradient can change in an abrupt fashion, thereby concealing a stationary point. To this end, they introduce a notion of $(\delta, \epsilon)$ stationarity, a weakening of $\epsilon$-stationarity; specifically, if $\mathbf{x}$ is $(\delta, \epsilon)$-stationary, then there exists a convex combination of gradients in a $\delta$-neighborhood of $\mathbf{x}$ that has norm at most $\epsilon$. However, this does not mean that $\mathbf{x}$ is $\delta$-close to an $\epsilon$-stationary point of $\mathbf{x}$ as noted by Shamir [18] since 
the convex hull might contain a small vector without any of the vectors being necessarily small. However, as Shamir observes that one needs to accept that the $(\delta, \epsilon)$-stationarity notion may have such pathologies. Instead, an alternative might lie in minimizing a smoothed function $\mathbb{E}_{\mathbf{u}}[f(\mathbf{x}+\delta \mathbf{u})]$ where $u$ is a suitably defined random variable. This avenue allows for leveraging a richer set of techniques but may still be afflicted by similar challenges.

We consider a zeroth-order smoothing approach in constrained, stochastic, and block-structured regimes with a view towards developing finite-time and asymptotic guarantees.

Contributions. We develop a randomized zeroth-order framework in regime where $f$ is block-structured and expectationvalued over $\mathcal{X}$, a Cartesian product of closed convex sets in which locally randomized smoothing is carried out via spherical smoothing. This scheme leads to a stochastic approximation framework in which the gradient estimator is constructed via (sampled) function values. Succinctly, our main contributions are captured as follows.

(i) Almost sure convergence guarantees. On applying the randomized scheme to an $\eta$-smoothed problem, under suitable choices of the steplength and mini-batch sequences, we show that the norm of the residual function of the smoothed problem tends to zero almost surely.

(ii) Rate and complexity guarantees. It can be shown that the expected squared residual (associated with the smoothed problem) diminishes at the rate of $\mathcal{O}(1 / k)$ in terms of projection steps on $\mathcal{X}_{i}$, leading to a complexity of $\mathcal{O}\left(\eta^{-2} \epsilon^{-4}\right)$ in terms of sampled function evaluations.

Notation. We use $\mathbf{x}, \mathbf{x}^{T}$, and $\|\mathbf{x}\|$ to denote a column vector, its transpose, and its Euclidean norm, respectively. We use $\mathbf{x}^{(i)}$ to denote the $i$ th block coordinate of vector $\mathbf{x}=$ $\left(\mathbf{x}^{(1)}, \ldots, \mathbf{x}^{(b)}\right)$. Given a mapping $F: \mathbb{R}^{n} \rightarrow \mathbb{R}^{n}, F_{i}$ denotes the $i$ th block coordinate of $F$. We define $f^{*} \triangleq \inf _{\mathbf{x} \in \mathcal{X}} f(\mathbf{x})$ and $f_{\eta}^{*} \triangleq \inf _{\mathbf{x} \in \mathcal{X}} f_{\eta}(\mathbf{x})$, where $f_{\eta}(\mathbf{x})$ denotes the smoothed approximation of $f$. Given a continuous function, i.e., $f \in$ $C^{0}$, we write $f \in C^{0,0}(\mathcal{X})$ if $f$ is Lipschitz continuous on the set $\mathcal{X}$ with parameter $L_{0}$. Given a continuously differentiable function, i.e., $f \in C^{1}$, we write $f \in C^{1,1}(\mathcal{X})$ if $\nabla f$ is Lipschitz continuous on $\mathcal{X}$ with parameter $L_{1}$. We write a.s. for "almost surely" and $\mathbb{E}[Z]$ denotes the expectation of a random variable $Z$. Given a scalar $u,[u]_{+} \triangleq \max \{0, u\}$.

\section{Stationarity AND SMOOTHING}

We first recap some concepts of Clarke's nonsmooth calculus [4] that allow for providing stationarity conditions. We first define the directional derivative, a key object necessary in addressing nonsmooth optimization problems.

Definition 1 (Directional derivatives and Clarke generalized gradient [4]). The directional derivative of $h$ at $\mathbf{x}$ in a direction $v$ is defined as

$$
h^{\circ}(\mathbf{x}, v) \triangleq \limsup _{\mathbf{y} \rightarrow \mathbf{x}, t \downarrow 0}\left(\frac{h(\mathbf{y}+t v)-h(\mathbf{y})}{t}\right) .
$$

The Clarke generalized gradient at $\mathbf{x}$ can then be defined as

$$
\partial h(\mathbf{x}) \triangleq\left\{\xi \in \mathbb{R}^{n} \mid h^{\circ}(\mathbf{x}, v) \geq \xi^{T} v, \quad \forall v \in \mathbb{R}^{n}\right\} .
$$

In other words, $h^{\circ}(\mathbf{x}, v)=\sup _{g \in \partial h(\mathbf{x})} g^{T} v$.

If $h$ is $C^{1}$ at $\mathbf{x}$, the Clarke generalized gradient reduces to the standard gradient, i.e. $\partial h(\mathbf{x})=\nabla_{\mathbf{x}} h(\mathbf{x})$. If $\mathbf{x}$ is a local minimizer of $h$, then we have that $0 \in \partial h(\mathbf{x})$. In fact, this claim can be extended to convex constrained regimes, i.e. if $\mathbf{x}$ is a local minimizer of $\min _{\mathbf{x} \in \mathcal{X}} h(\mathbf{x})$, then $\mathbf{x}$ satisfies $0 \in \partial h(\mathbf{x})+\mathcal{N}_{\mathcal{X}}(\mathbf{x})$, where $\mathcal{N}_{\mathcal{X}}(\mathbf{x})$ denotes the normal cone of $\mathcal{X}$ defined at $\mathrm{x}$ [4]. We now review some properties of $\partial h(\mathbf{x})$. In particular, if $h$ is locally Lipschitz on an open set $\mathcal{C}$ containing $\mathcal{X}$, then $h$ is differentiable almost everywhere on $\mathcal{C}$ by Rademacher's theorem [4]. Suppose $\mathcal{C}_{h}$ denotes the set of points where $h$ is not differentiable. We now provide some properties of the Clarke generalized gradient.

Proposition 1 (Properties of Clarke generalized gradients [4]). Suppose $h$ is $L_{0}$-Lipschitz continuous on $\mathbb{R}^{n}$. Then the following hold.

(i) $\partial h(\mathbf{x})$ is a nonempty, convex, and compact set and $\|g\| \leq L_{0}$ for any $g \in \partial h(\mathbf{x})$.

(ii) $h$ is differentiable almost everywhere.

(iii) $\partial h(\mathbf{x})$ is an upper semicontinuous map defined as

$\partial h(\mathbf{x}) \triangleq \operatorname{conv}\left\{g \mid g=\lim _{k \rightarrow \infty} \nabla_{\mathbf{x}} h\left(\mathbf{x}_{k}\right), \mathcal{C}_{h} \not \supset \mathbf{x}_{k} \rightarrow \mathbf{x}\right\}$.

We may also define the $\epsilon$-Clarke generalized gradient [11]

$$
\text { as } \partial_{\epsilon} h(\mathbf{x}) \triangleq \operatorname{conv}\{\xi: \xi \in \partial h(\mathbf{y}),\|\mathbf{x}-\mathbf{y}\| \leq \epsilon\} .
$$

When $f$ is nonsmooth and nonconvex on $\mathcal{X}$, a closed and convex set, then to contend with nonsmoothness, we consider a locally randomized smoothing technique described as follows. Given a function $h: \mathbb{R}^{n} \rightarrow \mathbb{R}$ and a scalar $\eta>0$, a smoothed approximation of $h$ is denoted by $h_{\eta}$ defined as

$$
h_{\eta}(\mathbf{x}) \triangleq \mathbb{E}_{u \in \mathbb{B}}[h(\mathbf{x}+\eta u)] .
$$

where $\mathbb{B} \triangleq\left\{u \in \mathbb{R}^{n} \mid\|u\| \leq 1\right\}$ denotes the unit ball and $u$ is uniformly distributed over $\mathbb{B}$. We now recall some properties of spherical smoothing. Throughout, $\mathbb{S} \triangleq\{v \in$ $\left.\mathbb{R}^{n} \mid\|v\|=1\right\}$ denotes the surface of $\mathbb{B}$ and $\mathcal{X}_{\eta} \triangleq \mathcal{X}+\eta \mathbb{B}$ represents the Minkowski sum of $\mathcal{X}$ and $\eta \mathbb{B}$.

Lemma 1 (Properties of spherical smoothing (cf. Lemma 1 in [5])). Suppose $h: \mathbb{R}^{n} \rightarrow \mathbb{R}$ is a continuous function and its smoothed counterpart $h_{\eta}$ is defined as (5), where $\eta>0$ is a given scalar. Then the following hold.

(i) $h_{\eta}$ is $C^{1}$ over $\mathcal{X}$ and

$$
\nabla_{\mathbf{x}} h_{\eta}(\mathbf{x})=\left(\frac{n}{\eta}\right) \mathbb{E}_{v \in \eta \mathbb{S}}\left[h(\mathbf{x}+v) \frac{v}{\|v\|}\right] \quad \forall \mathbf{x} \in \mathcal{X} .
$$

Suppose $h \in C^{0,0}\left(\mathcal{X}_{\eta}\right)$ with parameter $L_{0}$. For any $\mathbf{x}, \mathbf{y} \in$ $\mathcal{X}$, we have that (ii) - (iv) hold. 
(ii) $\left|h_{\eta}(\mathbf{x})-h_{\eta}(\mathbf{y})\right| \leq L_{0}\|\mathbf{x}-\mathbf{y}\|$.

(iii) $\left|h_{\eta}(\mathbf{x})-h(\mathbf{x})\right| \leq L_{0} \eta$.

(iv) $\left\|\nabla_{\mathbf{x}} h_{\eta}(\mathbf{x})-\nabla_{\mathbf{x}} h_{\eta}(\mathbf{y})\right\| \leq \frac{L_{0} n}{\eta}\|\mathbf{x}-\mathbf{y}\|$.

(v) Suppose $h \in C^{0,0}\left(\mathcal{X}_{\eta}\right)$ with parameter $L_{0}$ and

$$
g_{\eta}(\mathbf{x}, v) \triangleq\left(\frac{n}{\eta}\right) \frac{(h(\mathbf{x}+v)-h(\mathbf{x})) v}{\|v\|} .
$$

for $v \in \eta \mathbb{S}$. Then, for any $\mathbf{x} \in \mathcal{X}$, we have that $\mathbb{E}_{v \in \eta \mathbb{S}}\left[\left\|g_{\eta}(\mathbf{x}, v)\right\|^{2}\right] \leq L_{0}^{2} n^{2}$.

We restrict our attention to functions $f$ that are $L_{0^{-}}$ Lipschitz continuous over $\mathcal{X}_{\eta_{0}} \triangleq \mathcal{X}+\eta_{0} \mathbb{B}$. Further, we assume that $\tilde{f}(\mathbf{x}, \xi)-f(\mathbf{x})$ admits suitable bias and moment properties. We intend to develop schemes for computing approximate stationary points of (1) by an iterative scheme. However, we first need to formalizing the relationship between the original problem and its smoothed counterpart. This is provided in [5] by leveraging results from [15], [16].

Proposition 2 (Stationarity of $h_{\eta}$ and $\eta$-stationarity). Consider (1) where $f$ is a locally Lipschitz continuous function and $\mathcal{X}$ is a closed, convex, and bounded set in $\mathbb{R}^{n}$. (i) For any $\eta>0$ and any $\mathbf{x} \in \mathbb{R}^{n}, \nabla f_{\eta}(\mathbf{x}) \in \partial_{2 \eta} f(\mathbf{x})$. Furthermore, if $0 \notin \partial f(\mathbf{x})$, then there exists an $\eta$ such that $\nabla_{\mathbf{x}} f_{\tilde{\eta}}(\mathbf{x}) \neq 0$ for $\tilde{\eta} \in(0, \eta]$.

(ii) For any $\eta>0$ and any $\mathbf{x} \in \mathcal{X}$,

$$
0 \in \nabla_{\mathbf{x}} f_{\eta}(\mathbf{x})+\mathcal{N}_{\mathcal{X}}(\mathbf{x}) \Rightarrow 0 \in \partial_{2 \eta} f(\mathbf{x})+\mathcal{N}_{\mathcal{X}}(\mathbf{x}) .
$$

Intuitively, this means that if $\mathbf{x}$ is a stationary point of the $\eta$-smoothed problem, then $\mathbf{x}$ is a $2 \eta$-Clarke stationary point of the original problem. Next, we introduce a residual function that captures the departure from stationarity. Recall that when $h$ is a differentiable but possibly nonconvex function and $\mathcal{X}$ is a closed and convex set, then $\mathrm{x}$ is a stationary point of (1) if and only if

$$
G_{\beta}(\mathbf{x}) \triangleq \beta\left(\mathbf{x}-\Pi_{\mathcal{X}}\left[\mathbf{x}-\frac{1}{\beta} \nabla_{\mathbf{x}} f(\mathbf{x})\right]\right)=0 .
$$

When $f$ is not necessarily smooth as is the case in this paper, a residual of the smoothed problem can be derived by replacing $\nabla_{\mathbf{x}} f(\mathbf{x})$ by $\nabla_{\mathbf{x}} f_{\eta}(\mathbf{x})$. In particular, the residual $G_{\eta, \beta}$ denotes the stationarity residual with parameter $\beta$ of the $\eta$-smoothed problem while $\tilde{G}_{\eta, \beta}$ represents its counterpart arising from using a sampling-based estimate of $\nabla_{\mathbf{x}} f_{\eta}(\mathbf{x})$.

Definition 2 (The residual mapping). Suppose Assumption 1 holds. Given $\beta>0$ and a smoothing parameter $\eta>0$, for any $\mathbf{x} \in \mathbb{R}^{n}$ and $\tilde{e} \in \mathbb{R}^{n}$ an arbitrary given vector, let the residual mappings $G_{\eta, \beta}(\mathbf{x})$ and $\tilde{G}_{\eta, \beta}(\mathbf{x}, \tilde{e})$ be defined as

$$
\begin{aligned}
G_{\eta, \beta}(\mathbf{x}) & \triangleq \beta\left(\mathbf{x}-\Pi_{\mathcal{X}}\left[\mathbf{x}-\frac{1}{\beta} \nabla_{x} f_{\eta}(\mathbf{x})\right]\right) \text { and } \\
\tilde{G}_{\eta, \beta}(\mathbf{x}, \tilde{e}) & \triangleq \beta\left(\mathbf{x}-\Pi_{\mathcal{X}}\left[\mathbf{x}-\frac{1}{\beta}\left(\nabla_{x} f_{\eta}(\mathbf{x})+\tilde{e}\right)\right]\right) .
\end{aligned}
$$

Unsurprisingly, one can derive a bound on $\tilde{G}_{\eta, \beta}(\mathbf{x}, \tilde{e})$ in terms of $G_{\eta, \beta}(\mathbf{x})$ and $\tilde{e}$, as shown next.

Lemma 2 (Lemma 10 in [5]). Let Assumption 1 hold. Then the following holds for any $\beta>0, \eta>0$, and $\mathbf{x} \in \mathbb{R}^{n}$.

$$
\left\|G_{\eta, \beta}(\mathbf{x})\right\|^{2} \leq 2\left\|\tilde{G}_{\eta, \beta}(\mathbf{x}, \tilde{e})\right\|^{2}+2\|\tilde{e}\|^{2} .
$$

\section{A RANDOMIZED ZEROTH-ORDER ALGORITHM}

In this section, we provide the main assumptions, outline the proposed algorithm, and derive some preliminary results that will be utilized in the convergence analysis.

Assumption 1 (Problem properties). Consider problem (1).

(i) $f$ is $L_{0}$-Lipschitz on $\mathcal{X}+\eta_{0} \mathbb{B}$ for some $\eta_{0}>0$.

(ii) $\mathcal{X}_{i} \subseteq \mathbb{R}^{n}$ is a nonempty, closed, and convex set for $i=$ $1, \ldots, b$.

(iii) For all $\mathbf{x} \in \mathcal{X}_{\eta}+\eta_{0} \mathbb{S}$ we have $\mathbb{E}[\tilde{f}(\mathbf{x}, \omega) \mid \mathbf{x}]=f(\mathbf{x})$.

(iv) For all $\mathbf{x} \in \mathcal{X}_{\eta}+\eta_{0} \mathbb{S}$ we have $\mathbb{E}\left[\|\tilde{f}(\mathbf{x}, \omega)-f(\mathbf{x})\|^{2}\right.$ $\mid \mathbf{x}] \leq \nu^{2}$ for some $\nu>0$.

We introduce a variance-reduced randomized blockcoordinate zeroth-order scheme presented by Algorithm 1 To this end, we define a zeroth-order gradient estimate of $\tilde{f}\left(\mathbf{x}_{k}, \omega_{j, k}\right)$ as follows.

$g_{\eta}\left(\mathbf{x}_{k}, v_{j, k}, \omega_{j, k}\right) \triangleq \frac{n\left(\tilde{f}\left(\mathbf{x}_{k}+v_{j, k}, \omega_{j, k}\right)-\tilde{f}\left(\mathbf{x}_{k}, \omega_{j, k}\right)\right) v_{j, k}}{\left\|v_{j, k}\right\| \eta}$.

Intuitively, $g_{\eta}\left(\mathbf{x}_{k}, v_{j, k}, \omega_{j, k}\right)$ generates a gradient estimate by employing sampled function evaluations $\tilde{f}\left(\mathbf{x}_{k}, \omega_{j, k}\right)$ and $\tilde{f}\left(\mathbf{x}_{k}+v_{j, k}, \omega_{j, k}\right)$; in short, the zeroth-order oracle, given an $\mathbf{x}_{k}$ and a perturbation vector $v_{j, k}$, produces two evaluations, i.e. $\tilde{f}\left(\mathbf{x}_{k}, \omega_{j, k}\right)$ and $\tilde{f}\left(\mathbf{x}_{k}+v_{j, k}, \omega_{j, k}\right)$. We then employ a mini-batch approximation of this gradient estimate within a block coordinate (BC) structure. We formally define the stochastic errors emergent from the randomized $\mathrm{BC}$ scheme.

Definition 3. For all $k \geq 0$ and $j=1, \ldots, N_{k}$ we define

$$
\begin{aligned}
e_{j, k} & \triangleq \nabla \tilde{f}_{\eta}\left(\mathbf{x}_{k}, \omega_{j, k}\right)-\nabla f_{\eta}\left(\mathbf{x}_{k}\right), \\
\theta_{j, k} & \triangleq g_{\eta}\left(\mathbf{x}_{k}, v_{j, k}, \omega_{j, k}\right)-\nabla \tilde{f}_{\eta}\left(\mathbf{x}_{k}, \omega_{j, k}\right) \\
\delta_{j, k} & \triangleq b \mathbf{U}_{i_{k}} g_{\eta}\left(\mathbf{x}_{k}, v_{j, k}, \omega_{j, k}, i_{k}\right)-g_{\eta}\left(\mathbf{x}_{k}, v_{j, k}, \omega_{j, k}\right),
\end{aligned}
$$

where $e_{k} \triangleq \frac{\sum_{j=1}^{N_{k}} e_{j, k}}{N_{k}}, \theta_{k} \triangleq \frac{\sum_{j=1}^{N_{k}} \theta_{j, k}}{N_{k}}, \delta_{k} \triangleq \frac{\sum_{j=1}^{N_{k}} \delta_{j, k}}{N_{k}}$, and we define $\mathbf{U}_{\ell} \in \mathbb{R}^{n \times n_{\ell}}$ for $\ell \in\{1, \ldots, b\}$ such that $\left[\mathbf{U}_{1}, \ldots, \mathbf{U}_{b}\right]=\mathbf{I}_{n}$ and $\mathbf{I}_{n}$ denotes the $n \times n$ identity matrix.

The history of Algorithm 1 at iteration $k$ is defined as

$$
\mathcal{F}_{k} \triangleq \cup_{t=0}^{k-1}\left(\left\{i_{t}\right\} \cup\left(\cup_{j=1}^{N_{t}}\left\{\omega_{j, t}, v_{j, t}\right\}\right)\right), \quad \text { for } k \geq 1 .
$$

We impose the following independence requirement on $\omega_{j, k}, v_{j, k}$, and $i_{k}$. Recall that both $v_{j, k}$ and $i_{k}$ are user-defined so this is a mild requirement.

Assumption 2 (Independence ). Random samples $\omega_{j, k}, v_{j, k}$, and $i_{k}$ are generated independent of each other for all $k \geq 0$ and $1 \leq j \leq N_{k}$.

We now analyze the bias and moment properties of three crucial error sequences.

Lemma 3 (Bias and moment properties of $e, \delta$, and $\theta$ ). Consider Definition 3 Let Assumptions 1 and 2 hold. The following hold almost surely for $k \geq 0$ and $N_{k} \geq 1$.

(i) $\mathbb{E}\left[e_{j, k} \mid \mathcal{F}_{k}\right]=\mathbb{E}\left[\theta_{j, k} \mid \mathcal{F}_{k}\right]=\mathbb{E}\left[\delta_{j, k} \mid \mathcal{F}_{k}\right]=0$ almost surely for all $j=1, \ldots, N_{k}$. 


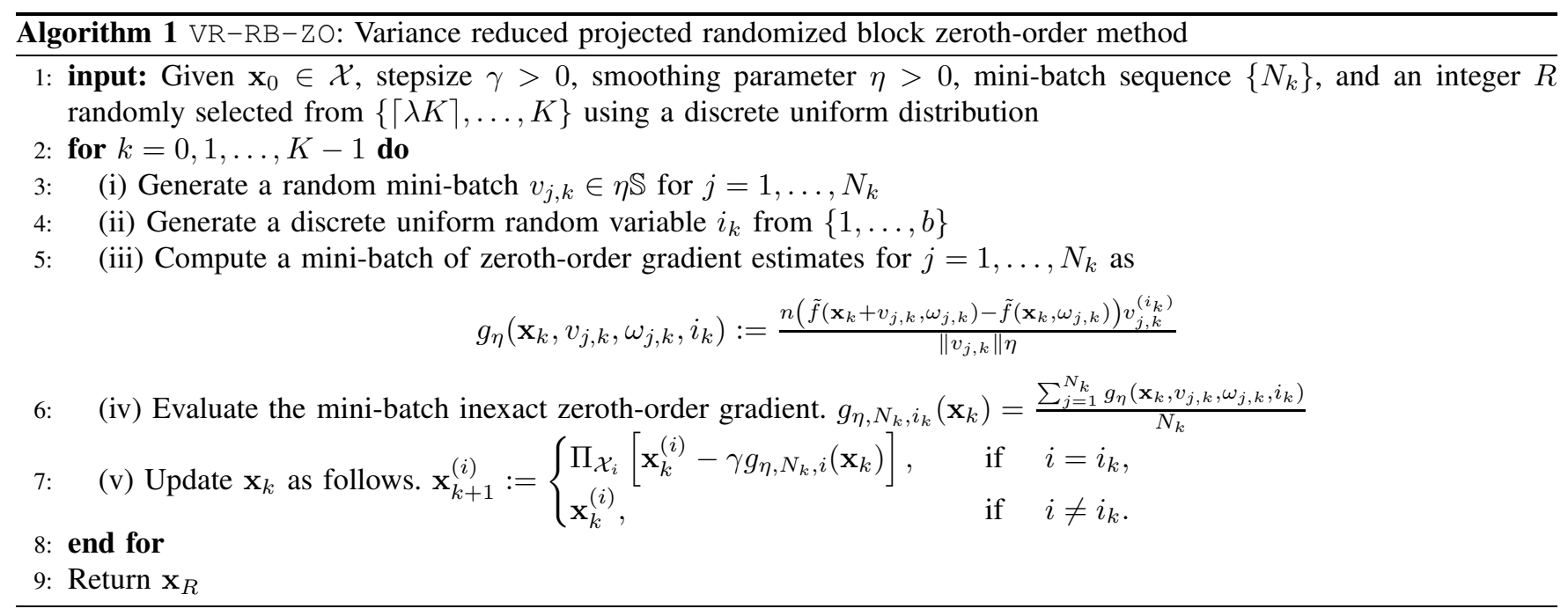

(ii) $\mathbb{E}\left[\left\|e_{k}\right\|^{2} \mid \mathcal{F}_{k}\right] \leq \frac{n^{2} \nu^{2}}{\eta^{2} N_{k}}, \mathbb{E}\left[\left\|\theta_{k}\right\|^{2} \mid \mathcal{F}_{k}\right] \leq \frac{L_{0}^{2} n^{2}}{N_{k}}$, and $\mathbb{E}\left[\left\|\delta_{k}\right\|^{2} \quad \mid \mathcal{F}_{k}\right] \leq \frac{3 n^{2}(b-1)}{N_{k}}\left(\frac{\nu^{2}}{\eta^{2}}+L_{0}^{2}+\left(\frac{\hat{f}}{\eta}\right)^{2}\right)$, almost surely where $\hat{f} \triangleq \sup _{\mathbf{x} \in \mathcal{X}+\eta \mathbb{S}} f(x)$.

Proof. (i) To show that $\mathbb{E}\left[e_{j, k} \mid \mathcal{F}_{k}\right]=0$ a.s., we may write

$$
\begin{aligned}
& \mathbb{E}\left[e_{j, k} \mid \mathcal{F}_{k}\right]=\mathbb{E}\left[\nabla \tilde{f}_{\eta}\left(\mathbf{x}_{k}, \omega_{j, k}\right)-\nabla f_{\eta}\left(\mathbf{x}_{k}\right) \mid \mathcal{F}_{k}\right] \\
& \stackrel{\text { Lemma }}{=} \frac{n}{\eta} \mathbb{E}\left[\mathbb{E}\left[\frac{\left(\tilde{f}\left(\mathbf{x}_{k}+v, \omega_{j, k}\right)-f\left(\mathbf{x}_{k}+v\right)\right) v}{\|v\|} \mid \mathcal{F}_{k} \cup\left\{\omega_{j, k}\right\}\right] \mid \mathcal{F}_{k}\right] \\
& \stackrel{\text { Assum. }}{=} \frac{n}{\eta} \mathbb{E}\left[\frac{\mathbb{E}\left[\tilde{f}\left(\mathbf{x}_{k}+v, \omega\right)-f\left(\mathbf{x}_{k}+v\right) \mid \mathcal{F}_{k} \cup\{v\}\right] v}{\|v\|} \mid \mathcal{F}_{k}\right] \\
& \stackrel{\text { Assum. }}{=} \frac{n}{\eta} \mathbb{E}\left[0 \times \frac{v}{\|v\|} \mid \mathcal{F}_{k}\right]=0, \quad \text { a.s. }
\end{aligned}
$$

To show that $\mathbb{E}\left[\theta_{j, k} \mid \mathcal{F}_{k}\right]=0$ a.s., we write

$$
\begin{aligned}
& \mathbb{E}\left[\theta_{j, k} \mid \mathcal{F}_{k}\right]=\mathbb{E}\left[g_{\eta}\left(\mathbf{x}_{k}, v_{j, k}, \omega_{j, k}\right)-\nabla \tilde{f}_{\eta}\left(\mathbf{x}_{k}, \omega_{j, k}\right) \mid \mathcal{F}_{k}\right] \\
& =\mathbb{E}\left[\frac{n\left(\tilde{f}\left(\mathbf{x}_{k}+v_{j, k}, \omega_{j, k}\right)-\tilde{f}\left(\mathbf{x}_{k}, \omega_{j, k}\right)\right) v_{j, k}}{\left\|v_{j, k}\right\| \eta}\right. \\
& \left.-\frac{n}{\eta} \mathbb{E}\left[\frac{\tilde{f}\left(\mathbf{x}_{k}+v, \omega_{j, k}\right) v}{\|v\|} \mid \mathcal{F}_{k} \cup\left\{\omega_{k, j}\right\}\right] \mid \mathcal{F}_{k}\right] \\
& \stackrel{\text { Assum. }}{=} \mathbb{E}\left[\mathbb{E}\left[\frac{n\left(\tilde{f}\left(\mathbf{x}_{k}+v_{j, k}, \omega_{j, k}\right)-\tilde{f}\left(\mathbf{x}_{k}, \omega_{j, k}\right)\right) v_{j, k}}{\left\|v_{j, k}\right\| \eta} \mid \mathcal{F}_{k} \cup\left\{v_{j, k}\right\}\right]\right. \\
& \left.-\frac{n}{\eta} \mathbb{E}\left[\tilde{f}\left(\mathbf{x}_{k}+v, \omega_{j, k}\right) \frac{v}{\|v\|} \mid \mathcal{F}_{k} \cup\{v\}\right] \mid \mathcal{F}_{k}\right] \\
& \stackrel{\text { Assum. }}{=} \mathbb{E}\left[\frac{n\left(f\left(\mathbf{x}_{k}+v_{j, k}\right)-f\left(\mathbf{x}_{k}\right)\right) v_{j, k}}{\left\|v_{j, k}\right\| \eta}-\frac{n}{\eta} f\left(\mathbf{x}_{k}+v\right) \frac{v}{\|v\|} \mid \mathcal{F}_{k}\right] \\
& =\frac{n}{\eta} \mathbb{E}_{v}\left[f\left(\mathbf{x}_{k}+v\right) \frac{v}{\|v\|}-f\left(\mathbf{x}_{k}+v\right) \frac{v}{\|v\|} \mid \mathbf{x}_{k}\right]=0,
\end{aligned}
$$

To show $\mathbb{E}\left[\delta_{j, k} \mid \mathcal{F}_{k}\right]=0$ a.s., we have almost surely

$$
\begin{aligned}
& \mathbb{E}\left[\delta_{j, k} \mid \mathcal{F}_{k}\right] \\
& =\mathbb{E}\left[b \mathbf{U}_{i_{k}} g_{\eta}\left(\mathbf{x}_{k}, v_{j, k}, \omega_{j, k}, i_{k}\right)-g_{\eta}\left(\mathbf{x}_{k}, v_{j, k}, \omega_{j, k}\right) \mid \mathcal{F}_{k}\right] \\
& \stackrel{\text { Assum. [ᄆ] }}{=} \mathbb{E}\left[\mathbb { E } \left[b \mathbf{U}_{i_{k}} g_{\eta}\left(\mathbf{x}_{k}, v_{j, k}, \omega_{j, k}, i_{k}\right)\right.\right. \\
& \left.\left.-g_{\eta}\left(\mathbf{x}_{k}, v_{j, k}, \omega_{j, k}\right) \mid \mathcal{F}_{k} \cup\left\{\omega_{j, k}, v_{j, k}\right\}\right] \mid \mathcal{F}_{k}\right] \\
& =\mathbb{E}\left[\sum_{i=1}^{b} b b^{-1} \mathbf{U}_{i} g_{\eta}\left(\mathbf{x}_{k}, v_{j, k}, \omega_{j, k}, i\right)-g_{\eta}\left(\mathbf{x}_{k}, v_{j, k}, \omega_{j, k}\right)\right. \\
& \left.\mid \mathcal{F}_{k}\right]=\mathbb{E}\left[g_{\eta}\left(\mathbf{x}_{k}, v_{j, k}, \omega_{j, k}\right)-g_{\eta}\left(\mathbf{x}_{k}, v_{j, k}, \omega_{j, k}\right) \mid \mathcal{F}_{k}\right]=0 .
\end{aligned}
$$

(ii) To show the first inequality, we may bound the conditional second moment of $e_{j, k}$ as follows.

$$
\begin{aligned}
& \mathbb{E}\left[\left\|e_{j, k}\right\|^{2} \mid \mathcal{F}_{k}\right]=\mathbb{E}\left[\left\|\nabla \tilde{f}_{\eta}\left(\mathbf{x}_{k}, \omega_{j, k}\right)-\nabla f_{\eta}\left(\mathbf{x}_{k}\right)\right\|^{2} \mid \mathcal{F}_{k}\right] \\
& \stackrel{\text { Lemma }}{=} \frac{n^{2}}{\eta^{2}} \mathbb{E}\left[\| \mathbb{E}\left[\tilde{f}\left(\mathbf{x}_{k}+v_{j, k}, \omega_{j, k}\right)-f\left(\mathbf{x}_{k}+v_{j, k}\right)\right.\right. \\
& \left.\left.\mid \mathcal{F}_{k} \cup\left\{\omega_{k, j}\right\}\right] \|^{2} \mid \mathcal{F}_{k}\right] \stackrel{\text { Jensen's ineq. }}{\leq} \frac{n^{2}}{\eta^{2}} \mathbb{E}\left[\mathbb { E } \left[\| \tilde{f}\left(\mathbf{x}_{k}+v_{j, k}, \omega_{j, k}\right)\right.\right. \\
& \left.\left.-f\left(\mathbf{x}_{k}+v_{j, k}\right) \|^{2} \mid \mathcal{F}_{k} \cup\left\{\omega_{k, j}\right\}\right] \mid \mathcal{F}_{k}\right] \\
& \stackrel{\text { Assumption! } \square}{\leq} \frac{n^{2}}{\eta^{2}} \mathbb{E}\left[\nu^{2} \mid \mathcal{F}_{k}\right]=\frac{n^{2} \nu^{2}}{\eta^{2}}, \quad \text { a.s. }
\end{aligned}
$$

From $\mathbb{E}\left[e_{j, k} \mid \mathcal{F}_{k}\right]=0$ a.s., Assumption 2 and Definition 3

$$
\begin{aligned}
& \mathbb{E}\left[\left\|e_{k}\right\|^{2} \mid \mathcal{F}_{k}\right]=\frac{1}{N_{k}^{2}} \mathbb{E}\left[\left\|\sum_{j=1}^{N_{k}} e_{j, k}\right\|^{2} \mid \mathcal{F}_{k}\right] \\
& =\frac{1}{N_{k}^{2}} \mathbb{E}\left[\sum_{j=1}^{N_{k}}\left\|e_{j, k}\right\|^{2} \mid \mathcal{F}_{k}\right]=\frac{1}{N_{k}^{2}} \sum_{j=1}^{N_{k}} \mathbb{E}\left[\left\|e_{j, k}\right\|^{2} \mid \mathcal{F}_{k}\right] .
\end{aligned}
$$

Combining the preceding two relations, we obtain $\mathbb{E}\left[\left\|e_{k}\right\|^{2}\right.$ $\left.\mathcal{F}_{k}\right] \leq \frac{n^{2} \nu^{2}}{\eta^{2} N_{k}}$ a.s. To show the second inequality, we have

$$
\begin{aligned}
& \mathbb{E}\left[\left\|\theta_{j, k}\right\|^{2} \mid \mathcal{F}_{k}\right] \\
& =\mathbb{E}\left[\left\|g_{\eta}\left(\mathbf{x}_{k}, v_{j, k}, \omega_{j, k}\right)-\nabla f_{\eta}\left(\mathbf{x}_{k}, \omega_{j, k}\right)\right\|^{2} \mid \mathcal{F}_{k}\right] \\
& \stackrel{\text { Assumption! }}{=} \mathbb{E}\left[\mathbb { E } \left[\left\|g_{\eta}\left(\mathbf{x}_{k}, v_{j, k}, \omega_{j, k}\right)-\nabla f_{\eta}\left(\mathbf{x}_{k}, \omega_{j, k}\right)\right\|^{2}\right.\right. \\
& \left.\left.\mid \mathcal{F}_{k} \cup\left\{\omega_{j, k}\right\}\right] \mid \mathcal{F}_{k}\right] \\
& \stackrel{\text { Lemma!(vii) }}{\leq} \mathbb{E}\left[L_{0}^{2} n^{2} \mid \mathcal{F}_{k}\right]=L_{0}^{2} n^{2}, \quad \text { a.s. }
\end{aligned}
$$

Similar to the proof of the first bound, we have that $\mathbb{E}\left[\left\|\theta_{k}\right\|^{2}\right.$ $\left.\mathcal{F}_{k}\right] \leq \frac{L_{0}^{2} n^{2}}{N_{k}}$ a.s. To derive the third bound, we may express

$$
\begin{aligned}
& \mathbb{E}\left[\left\|\delta_{j, k}\right\|^{2} \mid \mathcal{F}_{k}\right] \\
& =\mathbb{E}\left[\left\|b \mathbf{U}_{i_{k}} g_{\eta}\left(\mathbf{x}_{k}, v_{j, k}, \omega_{j, k}, i_{k}\right)-g_{\eta}\left(\mathbf{x}_{k}, v_{j, k}, \omega_{j, k}\right)\right\|^{2} \mid \mathcal{F}_{k}\right] \\
& \stackrel{\text { Assum. }}{=} \mathbb{E}\left[\mathbb { E } \left[\left\|b \mathbf{U}_{i_{k}} g_{\eta}\left(\mathbf{x}_{k}, v_{j, k}, \omega_{j, k}, i_{k}\right)-g_{\eta}\left(\mathbf{x}_{k}, v_{j, k}, \omega_{j, k}\right)\right\|^{2}\right.\right. \\
& \left.\left.\mid \mathcal{F}_{k} \cup\left\{\omega_{j, k}, v_{j, k}\right\}\right] \mid \mathcal{F}_{k}\right] \\
& =\mathbb{E}\left[\sum_{i=1}^{b} b^{-1}\left\|b \mathbf{U}_{i_{k}} g_{\eta}\left(\mathbf{x}_{k}, v_{j, k}, \omega_{j, k}, i\right)-g_{\eta}\left(\mathbf{x}_{k}, v_{j, k}, \omega_{j, k}\right)\right\|^{2}\right.
\end{aligned}
$$




$$
\begin{aligned}
& \left.\mid \mathcal{F}_{k}\right]=\mathbb{E}\left[\sum_{i=1}^{b} b\left\|\mathbf{U}_{i_{k}} g_{\eta}\left(\mathbf{x}_{k}, v_{j, k}, \omega_{j, k}, i\right)\right\|^{2}\right. \\
& +\left\|g_{\eta}\left(\mathbf{x}_{k}, v_{j, k}, \omega_{j, k}\right)\right\|^{2} \\
& \left.-2 g_{\eta}\left(\mathbf{x}_{k}, v_{j, k}, \omega_{j, k}\right)^{T} \sum_{i=1}^{b} \mathbf{U}_{i_{k}} g_{\eta}\left(\mathbf{x}_{k}, v_{j, k}, \omega_{j, k}, i\right) \mid \mathcal{F}_{k}\right] \\
& =\mathbb{E}\left[\sum_{i=1}^{b} b\left\|g_{\eta}\left(\mathbf{x}_{k}, v_{j, k}, \omega_{j, k}, i\right)\right\|^{2}-\left\|g_{\eta}\left(\mathbf{x}_{k}, v_{j, k}, \omega_{j, k}\right)\right\|^{2}\right. \\
& \left.\mid \mathcal{F}_{k}\right]=(b-1) \mathbb{E}\left[\left\|g_{\eta}\left(\mathbf{x}_{k}, v_{j, k}, \omega_{j, k}\right)\right\|^{2} \mid \mathcal{F}_{k}\right] .
\end{aligned}
$$

We can also write

$$
\begin{aligned}
& \mathbb{E}\left[\left\|g_{\eta}\left(\mathbf{x}_{k}, v_{j, k}, \omega_{j, k}\right)\right\|^{2} \mid \mathcal{F}_{k}\right] \\
& =\mathbb{E}\left[\left\|e_{j, k}+\theta_{j, k}+\nabla f_{\eta}\left(\mathbf{x}_{k}\right)\right\|^{2} \mid \mathcal{F}_{k}\right] \\
& \leq 3 \mathbb{E}\left[\left\|e_{j, k}\right\|^{2}+\left\|\theta_{j, k}\right\|^{2}+\left\|\nabla f_{\eta}\left(\mathbf{x}_{k}\right)\right\|^{2} \mid \mathcal{F}_{k}\right] .
\end{aligned}
$$

Note that from Lemma 1 we have

$$
\begin{aligned}
& \mathbb{E}\left[\left\|\nabla f_{\eta}\left(\mathbf{x}_{k}\right)\right\|^{2} \mid \mathcal{F}_{k}\right] \\
& \stackrel{\text { Jensen's }}{\leq}\left(\frac{n}{\eta}\right)^{2}\left\|\mathbb{E}\left[f\left(\mathbf{x}_{k}+v\right) \mid \mathcal{F}_{k}\right]\right\|^{2} \leq\left(\frac{n \hat{f}}{\eta}\right)^{2},
\end{aligned}
$$

where the second inequality follows from noting that $\sup _{\mathbf{x} \in \mathcal{X}+\eta \mathbb{S}} f(\mathbf{x}) \leq \hat{f}$. From $\mathbb{E}\left[\delta_{j, k} \mid \mathcal{F}_{k}\right]=0$ a.s., Assumption [2] and the definition of $\delta_{k}$, we have

$$
\begin{aligned}
& \mathbb{E}\left[\left\|\delta_{k}\right\|^{2} \mid \mathcal{F}_{k}\right]=\frac{1}{N_{k}^{2}} \mathbb{E}\left[\left\|\sum_{j=1}^{N_{k}} \delta_{j, k}\right\|^{2} \mid \mathcal{F}_{k}\right] \\
& =\frac{1}{N_{k}^{2}} \mathbb{E}\left[\sum_{j=1}^{N_{k}}\left\|\delta_{j, k}\right\|^{2} \mid \mathcal{F}_{k}\right]=\frac{1}{N_{k}^{2}} \sum_{j=1}^{N_{k}} \mathbb{E}\left[\left\|\delta_{j, k}\right\|^{2} \mid \mathcal{F}_{k}\right] .
\end{aligned}
$$

From the preceding four inequalities we obtain

$$
\begin{aligned}
& \mathbb{E}\left[\left\|\delta_{k}\right\|^{2} \mid \mathcal{F}_{k}\right] \leq 3(1-b)\left(\frac{1}{N_{k}^{2}} \sum_{j=1}^{N_{k}} \mathbb{E}\left[\left\|e_{j, k}\right\|^{2} \mid \mathcal{F}_{k}\right]\right. \\
& \left.+\frac{1}{N_{k}^{2}} \sum_{j=1}^{N_{k}} \mathbb{E}\left[\left\|\theta_{j, k}\right\|^{2} \mid \mathcal{F}_{k}\right]+\frac{1}{N_{k}^{2}} \sum_{j=1}^{N_{k}}\left(\frac{n \hat{f}}{\eta}\right)^{2}\right) \\
& \leq 3(b-1)\left(\frac{n^{2} \nu^{2}}{\eta^{2} N_{k}}+\frac{L_{0}^{2} n^{2}}{N_{k}}+\left(\frac{n \hat{f}}{\eta}\right)^{2} \frac{1}{N_{k}}\right) \\
& =\frac{3 n^{2}(b-1)}{N_{k}}\left(\frac{\nu^{2}}{\eta^{2}}+L_{0}^{2}+\left(\frac{\hat{f}}{\eta}\right)^{2}\right), \quad \text { a.s. }
\end{aligned}
$$

\section{CONVERGENCE AND RATE ANALYSIS}

In this section, we analyze the rate and complexity guarantees for the proposed zeroth-order randomized scheme. The reader may note that in the proposed block-coordinate framework, the $i$ th block is randomly selected. If this randomly selected block is denoted by $i_{k}$ at the $k$ th epoch, then a projected (inexact and smoothed) gradient step is taken with respect to this block, i.e.

$$
\mathbf{x}_{k+1}^{(i)}:=\Pi_{\mathcal{X}_{i}}\left[\mathbf{x}_{k}^{(i)}-\gamma g_{\eta, N_{k}, i}\left(\mathbf{x}_{k}\right)\right], \text { where } i=i_{k}
$$

while $\mathbf{x}_{k+1}^{(j)}=\mathbf{x}_{k}^{(j)}$ for $j \neq i_{k}$. The next Lemma shows that this step can be recast as a projected gradient step for the entire vector $\mathrm{x}$ with respect to the set $\mathcal{X}$. Similar results have been proven in [6], [12], [23].
Lemma 4. Consider Definition 3 and Algorithm 1 Then the update rule of $\mathbf{x}_{k}$ can be compactly characterized as

$$
\mathbf{x}_{k+1}=\Pi_{\mathcal{X}}\left[\mathbf{x}_{k}-b^{-1} \gamma\left(\nabla f_{\eta}\left(\mathbf{x}_{k}\right)+e_{k}+\theta_{k}+\delta_{k}\right)\right] .
$$

Proof. Invoking the definition of $\mathbf{U}_{i_{k}}$ and the Cartesian structure of $\mathcal{X}$, the update rule of $\mathbf{x}_{k}$ in Algorithm 1 can be written as

$$
\mathbf{x}_{k+1}=\Pi_{\mathcal{X}}\left[\mathbf{x}_{k}-\gamma \mathbf{U}_{i_{k}} g_{\eta, N_{k}, i_{k}}\left(\mathbf{x}_{k}\right)\right] .
$$

Summing relations (10), (11), and (12) we have for all $k \geq 0$ and $j=1, \ldots, N_{k}$ that

$$
e_{j, k}+\theta_{j, k}+\delta_{j, k}=b \mathbf{U}_{i_{k}} g_{\eta}\left(\mathbf{x}_{k}, v_{j, k}, \omega_{j, k}, i_{k}\right)-\nabla f_{\eta}\left(\mathbf{x}_{k}\right) .
$$

Summing over $j$ and dividing by $N_{k}$, we obtain for $k \geq 0$

$$
\begin{aligned}
& e_{k}+\theta_{k}+\delta_{k}=b \mathbf{U}_{i_{k}} \frac{\sum_{j=1}^{N_{k}} g_{\eta}\left(\mathbf{x}_{k}, v_{j, k}, \omega_{j, k}, i_{k}\right)}{N_{k}}-\nabla f_{\eta}\left(\mathbf{x}_{k}\right) \\
& =b \mathbf{U}_{i_{k}} g_{\eta, N_{k}, i_{k}}\left(\mathbf{x}_{k}\right)-\nabla f_{\eta}\left(\mathbf{x}_{k}\right) .
\end{aligned}
$$

From this relation and (13), we obtain the result.

Next, we derive a bound on the residual $\left\|G_{\eta, b / \gamma}\left(\mathbf{x}_{k}\right)\right\|^{2}$.

Lemma 5. Let Assumption 11 hold. Consider Definition (3). Suppose $\mathbf{x}_{k}$ is generated by Algorithm $\square$ where $\gamma \in\left(0, \frac{b \eta}{n L_{0}}\right)$ for $\eta>0$. Then for all $k \geq 0$ we have

$$
\begin{aligned}
& \left(1-\frac{n L_{0} \gamma}{b \eta}\right) \frac{\gamma}{4 b}\left\|G_{\eta, b / \gamma}\left(\mathbf{x}_{k}\right)\right\|^{2} \leq f_{\eta}\left(\mathbf{x}_{k}\right)-f_{\eta}\left(\mathbf{x}_{k+1}\right) \\
& +\left(1-\frac{n L_{0} \gamma}{2 b \eta}\right) \frac{\gamma}{b}\left\|e_{k}+\theta_{k}+\delta_{k}\right\|^{2} .
\end{aligned}
$$

Proof. From Lemma 1 (iv), the gradient mapping $\nabla f_{\eta}$ is Lipschitz continuous with the parameter $L_{\eta} \triangleq \frac{n L_{0}}{\eta}$. From the descent lemma,

$$
\begin{aligned}
& f_{\eta}\left(\mathbf{x}_{k+1}\right) \leq f_{\eta}\left(\mathbf{x}_{k}\right)+\nabla f_{\eta}\left(\mathbf{x}_{k}\right)^{T}\left(\mathbf{x}_{k+1}-\mathbf{x}_{k}\right) \\
& +\frac{L_{\eta}}{2}\left\|\mathbf{x}_{k+1}-\mathbf{x}_{k}\right\|^{2} \\
& =f_{\eta}\left(\mathbf{x}_{k}\right)+\left(\nabla f_{\eta}\left(\mathbf{x}_{k}\right)+e_{k}+\theta_{k}+\delta_{k}\right)^{T}\left(\mathbf{x}_{k+1}-\mathbf{x}_{k}\right) \\
& -\left(e_{k}+\theta_{k}+\delta_{k}\right)^{T}\left(\mathbf{x}_{k+1}-\mathbf{x}_{k}\right)+\frac{L_{\eta}}{2}\left\|\mathbf{x}_{k+1}-\mathbf{x}_{k}\right\|^{2} .
\end{aligned}
$$

Invoking the properties of the Euclidean projection and the Cartesian structure of $\mathcal{X}$, we have $\left(\mathbf{x}_{k}-\right.$ $\left.b^{-1} \gamma\left(\nabla f_{\eta}\left(\mathbf{x}_{k}\right)+e_{k}+\theta_{k}+\delta_{k}\right)-\mathbf{x}_{k+1}\right)^{T}\left(\mathbf{x}_{k}-\mathbf{x}_{k+1}\right) \leq 0$. This implies that

$$
\begin{aligned}
& \left(\nabla f_{\eta}\left(\mathbf{x}_{k}\right)+e_{k}+\theta_{k}+\delta_{k}\right)^{T}\left(\mathbf{x}_{k+1}-x_{k}\right) \\
& \leq-\frac{b}{\gamma}\left\|\mathbf{x}_{k+1}-\mathbf{x}_{k}\right\|^{2} .
\end{aligned}
$$

In addition, we may also express $\left(e_{k}+\theta_{k}+\delta_{k}\right)^{T}\left(\mathbf{x}_{k+1}-\right.$ $\mathbf{x}_{k}$ ) as follows.

$$
\begin{aligned}
& -\left(e_{k}+\theta_{k}+\delta_{k}\right)^{T}\left(\mathbf{x}_{k+1}-\mathbf{x}_{k}\right) \\
& \leq \frac{\gamma}{2 b}\left\|e_{k}+\theta_{k}+\delta_{k}\right\|^{2}+\frac{b}{2 \gamma}\left\|\mathbf{x}_{k+1}-\mathbf{x}_{k}\right\|^{2} .
\end{aligned}
$$

Combining the inequalities (15), (16), and (17) we obtain

$$
\begin{aligned}
f_{\eta}\left(\mathbf{x}_{k+1}\right) & \leq f_{\eta}\left(\mathbf{x}_{k}\right)+\left(-\frac{b}{2 \gamma}+\frac{L_{\eta}}{2}\right)\left\|\mathbf{x}_{k+1}-\mathbf{x}_{k}\right\|^{2} \\
& +\frac{\gamma}{2 b}\left\|e_{k}+\theta_{k}+\delta_{k}\right\|^{2}
\end{aligned}
$$


From Definition 2 we obtain

$$
\begin{aligned}
& f_{\eta}\left(\mathbf{x}_{k+1}\right) \\
\leq & f_{\eta}\left(\mathbf{x}_{k}\right)+\left(-\frac{b}{2 \gamma}+\frac{L_{\eta}}{2}\right) \frac{\gamma^{2}}{b^{2}}\left\|\tilde{G}_{\eta, b / \gamma}\left(\mathbf{x}_{k}, e_{k}+\theta_{k}+\delta_{k}\right)\right\|^{2} \\
+ & \frac{\gamma}{2 b}\left\|e_{k}+\theta_{k}+\delta_{k}\right\|^{2} \\
= & f_{\eta}\left(\mathbf{x}_{k}\right)-\left(1-\frac{L_{\eta} \gamma}{b}\right) \frac{\gamma}{2 b}\left\|\tilde{G}_{\eta, b / \gamma}\left(\mathbf{x}_{k}, e_{k}+\theta_{k}+\delta_{k}\right)\right\|^{2} \\
+ & \frac{\gamma}{2 b}\left\|e_{k}+\theta_{k}+\delta_{k}\right\|^{2} .
\end{aligned}
$$

Using Lemma 2 and by the requirement that $\gamma<\frac{b}{L_{\eta}}$,

$$
\begin{aligned}
f_{\eta}\left(\mathbf{x}_{k+1}\right) & \leq f_{\eta}\left(\mathbf{x}_{k}\right)-\left(1-\frac{L_{\eta} \gamma}{b}\right) \frac{\gamma}{4 b}\left\|G_{\eta, b / \gamma}\left(\mathbf{x}_{k}\right)\right\|^{2} \\
& +\left(\frac{\gamma}{2 b}+\left(1-\frac{L_{\eta} \gamma}{b}\right) \frac{\gamma}{2 b}\right)\left\|e_{k}+\theta_{k}+\delta_{k}\right\|^{2} .
\end{aligned}
$$

From the preceding relation, we obtain the result.

We now present an almost sure convergence guarantee for the sequence generated by Algorithm 1 by relying on the Robbins-Siegmund Lemma.

Proposition 3 (Asymptotic guarantees for Alg. 1). Consider Algorithm 1 Let Assumptions 1 and 2 hold and $N_{k}:=$ $(k+1)^{1+\delta}$ for $k \geq 0$ and $\delta>0$. Then the following hold.

(i) $\left\|G_{\eta, b / \gamma}\left(\mathbf{x}_{k}\right)\right\| \underset{k \rightarrow \infty}{\stackrel{a . s .}{\longrightarrow}} 0$.

(ii) Every limit point of $\left\{\mathbf{x}_{k}\right\}$ lies in the set of $2 \eta$-Clarke stationary points of (1) in an almost sure sense.

Proof. Let $f_{\eta}^{*} \triangleq \inf _{x \in \mathcal{X}} f_{\eta}(\mathbf{x})$. By taking conditional expectations with respect to $\mathcal{F}_{k}$ on the both sides of the inequality (14), we have

$$
\begin{aligned}
& \mathbb{E}\left[\left(f_{\eta}\left(\mathbf{x}_{k+1}\right)-f_{\eta}^{*}\right) \mid \mathcal{F}_{k}\right] \\
\leq & \left(f_{\eta}\left(\mathbf{x}_{k}\right)-f_{\eta}^{*}\right)-\left(1-\frac{L_{\eta} \gamma}{b}\right) \frac{\gamma}{4 b}\left\|G_{\eta, b / \gamma}\left(\mathbf{x}_{k}\right)\right\|^{2} \\
+ & \left(\frac{\gamma}{2 b}+\left(1-\frac{L_{\eta} \gamma}{b}\right) \frac{\gamma}{2 b}\right) \mathbb{E}\left[\left\|e_{k}+\theta_{k}+\delta_{k}\right\|^{2} \mid \mathcal{F}_{k}\right] \\
\leq & \left(f_{\eta}\left(\mathbf{x}_{k}\right)-f_{\eta}^{*}\right)-\left(1-\frac{L_{\eta} \gamma}{b}\right) \frac{\gamma}{4 b}\left\|G_{\eta, b / \gamma}\left(\mathbf{x}_{k}\right)\right\|^{2} \\
+ & \left(\frac{\gamma}{2 b}+\left(1-\frac{L_{\eta} \gamma}{b}\right) \frac{\gamma}{2 b}\right) \frac{c}{N_{k}} .
\end{aligned}
$$

By the Robbins-Siegmund Lemma, the summability of $\left\|e_{k}+\theta_{k}+\delta_{k}\right\|^{2}$ (which holds by choice of $N_{k}$ ), and the nonnegativity of $f_{\eta}\left(\mathbf{x}_{k}\right)-f_{\eta}^{*}$, we have that $\left\{\left(f_{\eta}\left(\mathbf{x}_{k}\right)-\right.\right.$ $\left.\left.f_{\eta}^{*}\right)\right\}$ is convergent a.s. and $\sum_{k=1}^{\infty}\left\|G_{\eta, b / \gamma}\left(\mathbf{x}_{k}\right)\right\|^{2}<\infty$ almost surely. It remains to show that with probability one, $\left\|G_{\eta, b / \gamma}\left(\mathbf{x}_{k}\right)\right\| \rightarrow 0$ as $k \rightarrow \infty$. We proceed by contradiction. Suppose for $\omega \in \Omega_{1} \subset \Omega$ and $\mu\left(\Omega_{1}\right)>0$ (i.e. with finite probability), $\left\|G_{\eta, b / \gamma}\left(\mathbf{x}_{k}\right)\right\| \stackrel{k \in \mathcal{K}(\omega)}{\longrightarrow} \epsilon(\omega)>0$ where $\mathcal{K}(\omega)$ is a random subsequence. Consequently, for every $\omega \in \Omega_{1}$ and $\tilde{\delta}>0$, there exists $K(\omega)$ such that $k \geq K(\omega),\left\|G_{\eta, b / \gamma}\left(\mathbf{x}_{k}\right)\right\| \geq \frac{\epsilon(\omega)}{2}$. Consequently, we have that $\sum_{k \rightarrow \infty}\left\|G_{\eta, b / \gamma}\left(\mathbf{x}_{k}\right)\right\|^{2} \geq \sum_{k \in \mathcal{K}(\omega)}\left\|G_{\eta, b / \gamma}\left(\mathbf{x}_{k}\right)\right\|^{2} \geq$ $\sum_{k \in \mathcal{K}(\omega), k \geq K(\omega)}\left\|G_{\eta, b / \gamma}\left(\mathbf{x}_{k}\right)\right\|^{2}=\infty$ with finite probability. But this leads to a contradiction, implying that $\left\|G_{\eta, b / \gamma}\left(\mathbf{x}_{k}\right)\right\|^{2} \underset{k \rightarrow \infty}{\stackrel{a . s .}{\longrightarrow}} 0$.

(ii) Recall from Proposition 2 that if $\mathbf{x}$ satisfies $G_{\eta, b / \gamma}(\mathbf{x})=$ 0 , it is a $2 \eta$-Clarke stationary point of $(1)$, i.e. $0 \in \partial_{2 \eta} f(\mathbf{x})+$
$\mathcal{N}_{\mathcal{X}}(\mathbf{x})$. Since almost every limit point of $\left\{\mathbf{x}_{k}\right\}$ satisfies $G_{\eta, b / \gamma}(\mathbf{x})=0$, the result follows.

We now conclude this section with a formal rate statement and complexity guarantees in terms of projection steps on $\mathcal{X}_{i}$ as well as sampled function evaluations.

Theorem 1 (Rate and complexity statements for Alg. 1). Consider Algorithm 11 Let Assumptions 11 and 2 hold and $N_{k}:=\left\lceil 1+\frac{k+1}{\eta^{a}}\right\rceil$ for $k \geq 0$ and for some $a \geq 0$.

(i) For $\gamma<\frac{b \eta}{n L_{0}}$ and all $K>\frac{2}{1-\lambda}$ with $\ell \triangleq\lceil\lambda K\rceil$ we have

$$
\begin{aligned}
& \mathbb{E}\left[\left\|G_{\eta, b / \gamma}\left(\mathbf{x}_{R}\right)\right\|^{2}\right] \leq\left(\left(1-\frac{n L_{0} \gamma}{b \eta}\right) \frac{\gamma}{4 b}(1-\lambda) K\right)^{-1} \\
& \left(\mathbb{E}\left[f\left(\mathbf{x}_{\ell}\right)\right]-f^{*}+2 L_{0} \eta+3 n^{2}\left((3 b-2)\left(\nu^{2}+L_{0}^{2} \eta^{2}\right)\right.\right. \\
& \left.\left.+3(b-1) \hat{f}^{2}\right)(0.5-\ln (\lambda)) \eta^{2-a}\right) .
\end{aligned}
$$

(ii) Suppose $\gamma=\frac{b \eta}{2 n L_{0}}$. Let $\epsilon>0$ be an arbitrary scalar and $K_{\epsilon}$ be such that $\mathbb{E}\left[\left\|G_{\eta, b / \gamma}\left(\mathbf{x}_{R}\right)\right\|\right] \leq \epsilon$. Then:

(ii-1) The total number of projection steps on component sets is $K_{\epsilon}=\mathcal{O}\left(\eta^{-1+[a-2]+} \epsilon^{-2}\right)$.

(ii-2) The total sample complexity is $\mathcal{O}\left(\eta^{-a-2+2[a-2]}+\epsilon^{-4}\right)$.

Proof. (i) Consider the inequality of Lemma 2 . Summing from $k=\ell, \ldots, K-1$ where $\ell \triangleq\lceil\lambda K\rceil$ we have $\left(1-\frac{n L_{0} \gamma}{b \eta}\right) \frac{\gamma}{4 b} \sum_{k=\ell}^{K-1}\left\|G_{\eta, b / \gamma}\left(\mathbf{x}_{k}\right)\right\|^{2} \leq f_{\eta}\left(\mathbf{x}_{\ell}\right)-f_{\eta}\left(\mathbf{x}_{K}\right)+$ $\left(1-\frac{n L_{0} \gamma}{2 b \eta}\right) \frac{\gamma}{b} \sum_{k=\ell}^{K-1}\left\|e_{k}+\theta_{k}+\delta_{k}\right\|^{2}$. Taking expectations on both sides, we obtain

$$
\begin{gathered}
\left(1-\frac{n L_{0} \gamma}{b \eta}\right) \frac{\gamma}{4 b}(K-\ell) \mathbb{E}\left[\left\|G_{\eta, b / \gamma}\left(\mathbf{x}_{R}\right)\right\|^{2}\right] \leq \\
\left(1-\frac{n L_{0} \gamma}{2 b \eta}\right) \frac{\gamma}{b} \sum_{k=\ell}^{K-1} \mathbb{E}\left[\left\|e_{k}+\theta_{k}+\delta_{k}\right\|^{2}\right]+\mathbb{E}\left[f_{\eta}\left(\mathbf{x}_{\ell}\right)\right]-f_{\eta}^{*} .
\end{gathered}
$$

The last term on the right can be expressed as

$$
\begin{aligned}
& \mathbb{E}\left[f_{\eta}\left(\mathbf{x}_{\ell}\right)\right]-f_{\eta}^{*}=\mathbb{E}\left[f\left(\mathbf{x}_{\ell}\right)+f_{\eta}\left(\mathbf{x}_{\ell}\right)-f\left(\mathbf{x}_{\ell}\right)\right]-f_{\eta}^{*}+f^{*} \\
& -f^{*}=\mathbb{E}\left[f\left(\mathbf{x}_{\ell}\right)\right]-f^{*}+\mathbb{E}\left[\left|f_{\eta}\left(\mathbf{x}_{\ell}\right)-f\left(\mathbf{x}_{\ell}\right)\right|\right]+\left|f^{*}-f_{\eta}^{*}\right| \\
& \stackrel{\text { Lemma (耳ii) }}{\leq} \mathbb{E}\left[f\left(\mathbf{x}_{\ell}\right)\right]-f^{*}+2 L_{0} \eta .
\end{aligned}
$$

From Lemma 3 (ii), we obtain

$$
\begin{aligned}
& \mathbb{E}\left[\left\|e_{k}+\theta_{k}+\delta_{k}\right\|^{2}\right] \leq 3 \mathbb{E}\left[\mathbb{E}\left[\left\|e_{k}\right\|^{2}+\left\|\theta_{k}\right\|^{2}+\left\|\delta_{k}\right\|^{2} \mid \mathcal{F}_{k}\right]\right] \\
& \leq \frac{3 n^{2} \nu^{2}}{\eta^{2} N_{k}}+\frac{3 L_{0}^{2} n^{2}}{N_{k}}+\frac{9 n^{2}(b-1)}{N_{k}}\left(\frac{\nu^{2}}{\eta^{2}}+L_{0}^{2}+\left(\frac{\hat{f}}{\eta}\right)^{2}\right) \\
& \leq \frac{3 n^{2}}{N_{k}}\left(\frac{(3 b-2) \nu^{2}}{\eta^{2}}+(3 b-2) L_{0}^{2}+3(b-1)\left(\frac{\hat{f}}{\eta}\right)^{2}\right) .
\end{aligned}
$$


From the preceding relations we obtain

$$
\begin{aligned}
& \mathbb{E}\left[\left\|G_{\eta, b / \gamma}\left(\mathbf{x}_{R}\right)\right\|^{2}\right] \leq\left(\left(1-\frac{n L_{0} \gamma}{b \eta}\right) \frac{\gamma}{4 b}(K-\ell)\right)^{-1} \times \\
& \left(\mathbb{E}\left[f\left(\mathbf{x}_{\ell}\right)\right]-f^{*}+2 L_{0} \eta+\left((3 b-2) \nu^{2}+(3 b-2) L_{0}^{2} \eta^{2}\right.\right. \\
& \left.\left.+3(b-1)(\hat{f})^{2}\right) \sum_{k=\ell}^{K-1} \frac{3 n^{2}}{\eta^{2} N_{k}}\right) \\
& \leq\left(\left(1-\frac{n L_{0} \gamma}{b \eta}\right) \frac{\gamma}{4 b}(K-\ell)\right)^{-1} \times \\
& \left(\mathbb{E}\left[f\left(\mathbf{x}_{\ell}\right)\right]-f^{*}+2 L_{0} \eta+\left((3 b-2) \nu^{2}+(3 b-2) L_{0}^{2} \eta^{2}\right.\right. \\
& \left.\left.+3(b-1)(\hat{f})^{2}\right) \sum_{k=\ell}^{K-1} \frac{3 n^{2}}{\eta^{2-a} k+1}\right),
\end{aligned}
$$

where the last inequality is a consequence of $N_{k}:=$ $\left\lceil 1+\frac{k+1}{\eta^{a}}\right\rceil \geq \frac{k+1}{\eta^{a}}$. Recall that $K>\frac{2}{1-\lambda}$ implies $\ell \leq K-1$ and $\sum_{k=\ell}^{K-1} \frac{1}{k+1} \leq 0.5+\ln \left(\frac{N}{\lambda N+1}\right) \leq 0.5-\ln (\lambda)$. Further, $K-\ell \geq K-\lambda K=(1-\lambda) K$ implying that

$$
\begin{aligned}
& \mathbb{E}\left[\left\|G_{\eta, b / \gamma}\left(\mathbf{x}_{R}\right)\right\|^{2}\right] \leq\left(\left(1-\frac{n L_{0} \gamma}{b \eta}\right) \frac{\gamma}{4 b}(1-\lambda) K\right)^{-1} \times \\
& \left(\mathbb{E}\left[f\left(\mathbf{x}_{\ell}\right)\right]-f^{*}+2 L_{0} \eta+3 n^{2}\left((3 b-2) \nu^{2}+(3 b-2) L_{0}^{2} \eta^{2}\right.\right. \\
& \left.\left.+3(b-1) \hat{f}^{2}\right)(0.5-\ln (\lambda)) \eta^{a-2}\right) .
\end{aligned}
$$

(ii) To show (ii-1), using the relation in part (i) and substituting $\gamma:=\frac{b \eta}{2 n L_{0}}$ we obtain

$$
\begin{aligned}
& \mathbb{E}\left[\left\|G_{\eta, b / \gamma}\left(\mathbf{x}_{R}\right)\right\|^{2}\right] \leq\left(16 n L_{0}\right)(\eta(1-\lambda) K)^{-1} \times \\
& \left(\mathbb{E}\left[f\left(\mathbf{x}_{\ell}\right)\right]-f^{*}+2 L_{0} \eta+3 n^{2}\left((3 b-2) \nu^{2}+(3 b-2) L_{0}^{2} \eta^{2}\right.\right. \\
& \left.\left.+3(b-1) \hat{f}^{2}\right)(0.5-\ln (\lambda)) \eta^{a-2}\right) .
\end{aligned}
$$

From Jensen's inequality, it follows that $\mathbb{E}\left[\left\|G_{\eta, 1 / \gamma}\left(\mathbf{x}_{R}\right)\right\|\right] \leq \sqrt{\mathcal{O}\left(\eta^{\left.-1+[a-2]+K^{-1}\right)}\right.}$ and thus, we have $K_{\epsilon}=\mathcal{O}\left(\eta^{-1+[a-2]+} \epsilon^{-2}\right)$. Next, we show (ii-2). The total sample complexity of upper-level is as follows.

$$
\begin{aligned}
\sum_{k=0}^{K_{\epsilon}} N_{k} & =\sum_{k=0}^{K_{\epsilon}}\left\lceil 1+\frac{(k+1)}{\eta^{a}}\right\rceil \leq \mathcal{O}\left(K_{\epsilon}\right)+\mathcal{O}\left(\frac{K_{\epsilon}^{2}}{\eta^{a}}\right) \\
& \leq \mathcal{O}\left(\eta^{-a-2+2[a-2]}+\epsilon^{-4}\right) .
\end{aligned}
$$

Remark 1. (i) For $0 \leq a<2$, we attain the best iteration complexity of $\mathcal{O}\left(\eta^{-1} \epsilon^{-2}\right)$ and sample complexity of $\mathcal{O}\left(\eta^{-2} \epsilon^{-4}\right)$ in terms of dependence of $\eta$ when $a=0$. (ii) When $a \geq 2$, these statements change to $\mathcal{O}\left(\eta^{a-3} \epsilon^{-2}\right)$ and $\mathcal{O}\left(\eta^{a-6} \epsilon^{-4}\right)$, respectively. Within this range, for $a:=6$ we obtain a sample complexity of $\mathcal{O}\left(\epsilon^{-4}\right)$ that is invariant in terms of $\eta$, but a small $\eta$ leads to a large batch size making the implementation of the scheme less appealing. Exploring these trade-offs remains a future direction to our research. (iii) Our results are comparable to those obtained in [24], which uses neither smoothing nor a zeroth-order framework.

\section{CONCLUding REMARKS}

While a significant amount of prior research has analyzed nonsmooth and nonconvex optimization problems, much of this effort has relied on either the imposition of structural assumptions on the problem or required weak convexity, rather than general nonconvexity. Little research, if any, is available in stochastic regimes to contend with general nonconvex and nonsmooth optimization problems. To this end, we develop a randomized smoothing framework which allows for claiming that a stationary point of the $\eta$-smoothed problem is a $2 \eta$-stationary point for the original problem in the Clarke sense. By utilizing a suitable residual function that provides a metric for stationarity for the smoothed problem, we present a zeroth-order framework reliant on utilizing sampled function evaluations implemented in a block-structured regime. In this setting, we make two sets of contributions for the sequence generated by the proposed scheme. (i) The residual function of the smoothed problem tends to zero almost surely along the generated sequence; (ii) To compute an $\mathbf{x}$ that ensures that the expected norm of the residual of the $\eta$-smoothed problem is within $\epsilon$, we proceed to show that no more than $\mathcal{O}\left(\eta^{-1} \epsilon^{-2}\right)$ projection steps and $\mathcal{O}\left(\eta^{-2} \epsilon^{-4}\right)$ function evaluations are required.

\section{REFERENCES}

[1] J. V. Burke, A. S. Lewis, and M. L. Overton. Approximating subdifferentials by random sampling of gradients. Math. Oper. Res., 27(3):567-584, 2002.

[2] J. V. Burke, A. S. Lewis, and M. L. Overton. A robust gradient sampling algorithm for nonsmooth, nonconvex optimization. SIAM J. Optim., 15(3):751-779, 2005.

[3] X. Chen. Smoothing methods for nonsmooth, nonconvex minimization. Math. Program., 134(1, Ser. B):71-99, 2012.

[4] F. H. Clarke, Yu. S. Ledyaev, R. J. Stern, and P. R. Wolenski. Nonsmooth analysis and control theory, volume 178 of Graduate Texts in Mathematics. Springer-Verlag, New York, 1998.

[5] S. Cui, U. V. Shanbhag, and F. Yousefian. Complexity guarantees for an implicit smoothing-enabled method for stochastic MPECs. under review, 2021. https://arxiv.org/abs/2104.08406

[6] C. D. Dang and G. Lan. Stochastic block mirror descent methods for nonsmooth and stochastic optimization. SIAM Journal on Optimization, 25(2):856-881, 2015.

[7] D. Davis and D. Drusvyatskiy. Stochastic model-based minimization of weakly convex functions. SIAM J. Optim., 29(1):207-239, 2019.

[8] D. Davis and B. Grimmer. Proximally guided stochastic subgradient method for nonsmooth, nonconvex problems. SIAM J. Optim., 29(3):1908-1930, 2019.

[9] Y. M. Ermoliev, V. I. Norkin, and R. J.-B. Wets. The minimization of semicontinuous functions: mollifier subgradients. SIAM J. Control Optim., 33(1):149-167, 1995.

[10] S. Ghadimi, G. Lan, and H. Zhang. Mini-batch stochastic approximation methods for nonconvex stochastic composite optimization. Math. Programming, 155(1-2):267-305, 2016.

[11] A. A. Goldstein. Optimization of Lipschitz continuous functions. Math. Programming, 13(1):14-22, 1977.

[12] H. D. Kaushik and F. Yousefian. A method with convergence rates for optimization problems with variational inequality constraints. SIAM Journal on Optimization (accepted), 2021. https://arxiv.org/abs/2007.15845

[13] K. C. Kiwiel. Convergence of the gradient sampling algorithm for nonsmooth nonconvex optimization. SIAM J. Optim., 18(2):379-388, 2007.

[14] J. Lei and U. V. Shanbhag. Asynchronous variance-reduced block schemes for composite non-convex stochastic optimization: blockspecific steplengths and adapted batch-sizes. Optimization Methods and Software, pages 1-31, 2020.

[15] D. Q. Mayne and E. Polak. Nondifferential optimization via adaptive smoothing. J. Optim. Theory Appl., 43(4):601-613, 1984.

[16] BS Mordukhovich. Variational analysis and generalized differentiation. i. basic theory, ii. applications., 2009.

[17] Y. Nesterov and V. Spokoiny. Random gradient-free minimization of convex functions. Found. Comput. Math., 17(2):527-566, 2017. 
[18] O. Shamir. Can we find near-approximately-stationary points of nonsmooth nonconvex functions? In OPT2020: 12th Annual Workshop on Optimization for Machine Learning, 2021.

[19] A. Shapiro, D. Dentcheva, and A. Ruszczyński. Lectures on stochastic programming, volume 9 of MPS/SIAM Series on Optimization. SIAM, Philadelphia, PA, 2009. Modeling and theory.

[20] V. A. Steklov. Sur les expressions asymptotiques decertaines fonctions définies par les équations différentielles du second ordre et leers applications au problème du dévelopement d'une fonction arbitraire en séries procédant suivant les diverses fonctions. Comm. Charkov Math. Soc., 2(10):97-199, 1907.

[21] Y. Xu and W. Yin. A globally convergent algorithm for nonconvex optimization based on block coordinate update. Journal of Scientific Computing, pages 1-35, 2017.

[22] F. Yousefian, A. Nedić, and U. V. Shanbhag. On stochastic gradient and subgradient methods with adaptive steplength sequences. Automatica, 48(1):56-67, 2012.

[23] F. Yousefian, A. Nedić, and U. V. Shanbhag. On stochastic mirror-prox algorithms for stochastic Cartesian variational inequalities: Randomized block coordinate and optimal averaging schemes. Set-Valued and Variational Analysis, 26(4):789-819, 2018.

[24] J. Zhang, H. Lin, S. Jegelka, S. Sra, and A. Jadbabaie. Complexity of finding stationary points of nonconvex nonsmooth functions. In Proceedings of the 37th International Conference on Machine Learning, volume 119, pages 11173-11182. PMLR, 2020. 